\title{
Determination of the Optical Absorption Coefficient Spectra of Thin Semiconductor Layers from Their Photoacoustic Spectra
}

\author{
L. Bychto ${ }^{1}$ - M. Maliński ${ }^{1}$
}

Received: 9 November 2017 / Accepted: 24 July 2018 / Published online: 1 August 2018

(c) The Author(s) 2018

\begin{abstract}
This paper presents the new method of computation of the optical absorption coefficient spectra from the normalized photoacoustic amplitude spectra of thin semiconductor samples deposited on the optically transparent and thermally thick substrates. The simple equation for computations of the optical absorption coefficient spectrum from the normalized photoacoustic amplitude spectrum is derived and discussed. This model applied for computations of the optical absorption coefficient spectrum of thin germanium samples, deposited on the transparent glass substrate, from their photoacoustic spectra proved its correctness and usefulness.
\end{abstract}

Keywords Optical absorption coefficient spectra $\cdot$ Photoacoustics $\cdot$ Photothermal spectroscopy · Thin semiconductor films

\section{Introduction}

The photoacoustic spectroscopy is often used for investigations of optical properties of thick and thin semiconducting layers. A lot of papers describe PA spectra of thin solar cells materials of the type: CuInGaSe [1, 2], CuInSe [3, 4], CdTe [5], CdS [6, 7], $\mathrm{CdZnS}[8]$ and $\mathrm{In}_{2} \mathrm{~S}_{3}$ [9].

Some PA results concern investigations of thin SiC layers [10], or other thin layers [11]. A lot of papers describe PA spectra characteristics of thin porous layers on silicon [12]. Many papers describe PA spectra of thick semiconductor samples especially of mixed crystals $[13,14]$. PA spectroscopy is also used for investigations of differently treated surfaces of silicon samples [15].

\footnotetext{
$凶$ L. Bychto

leszek.bychto@tu.koszalin.pl

1 Faculty of Electronics and Computer Science, Koszalin University of Technology, 2 Sniadeckich St, 75-453 Koszalin, Poland
} 
The problem with the application of the photoacoustic spectroscopy method, for investigations of thin layers, is that for small values of the optical absorption coefficient, the influence of multiple reflections of light should be taken into account. This problem was analyzed theoretically and experimentally, among others, in papers [16-18]. In these papers, the mathematical equations for the photoacoustic signals in the presence of the reflections of light are presented and discussed. The expression for the photoacoustic signal of the samples in the presence of the multiple reflections of light was also presented in paper [19].

The main goal of photoacoustic spectroscopic investigations of thin layers presented in the literature [1-9] was to determine the energy gap of thin semiconductor layers or their optical absorption coefficient spectrum, and to extract their optical parameters depending on the technological parameters of deposition of the layers.

The goal of this paper is to present a new method of computation of the optical absorption coefficient spectra from the photoacoustic amplitude spectra. It can be applied for thermally thin semiconductor layers deposited on the optically transparent and thermally thick substrates. The two cases are analyzed: when the optical reflection coefficient $R_{\text {opt }}$ can be neglected and when it cannot be neglected. The influence of the value of this parameter on the computed optical absorption coefficient spectra is also presented and discussed.

\section{Theoretical Model}

For the thermally and optically thick samples, the optical absorption coefficient spectrum can be computed from the normalized photoacoustic amplitude spectrum $q$ [20-22] by Eq. 1.

$$
\beta=\frac{1}{\mu} \cdot \frac{q^{2}+q \cdot \sqrt{2-q^{2}}}{1-q^{2}}
$$

where $\beta$ is the optical absorption coefficient, $\mu=(\alpha / \pi \cdot f)^{1 / 2}$ is the thermal diffusion length, $\alpha$ is the thermal diffusivity, $f$ is the frequency of modulation of the intensity of light.

For thin samples, the formulae for computations of the optical absorption coefficient spectrum from the normalized photoacoustic amplitude spectrum $q$, in two specific cases, were derived and are presented below.

\subsection{Multiple Reflections of Light in the Sample can be Neglected}

The temperature of the front (illuminated) surface of the sample on the optically transparent and thermally thick substrate can be described by Eq. 2. It was derived in paper [23].

$$
T_{F}=\frac{\left(1-R_{\mathrm{opt}}\right) \beta}{\lambda \sigma(1-R \cdot \exp (-2 \sigma d))}\left(\frac{1-\exp (-(\beta+\sigma) d)}{\beta+\sigma}+\frac{R \cdot \exp (-2 \sigma d) \cdot(1-\exp (-(\beta-\sigma) d))}{\beta-\sigma}\right)
$$


where $d$ is the thickness of the sample, $\sigma$ is the parameter defined as $\sigma=(1+\mathrm{i}) / \mu, \lambda$ is the thermal conductivity, $R=\left(e_{s}-e_{b}\right) /\left(e_{s}+e_{b}\right)$ is the thermal reflection coefficient between the sample and the backing, and $e_{\mathrm{s}}$ and $e_{b}$ are the thermal effusivities of the sample and the glass backing, respectively. $R_{\mathrm{opt}}$ is the optical reflection coefficient of the sample.

For a large value of the optical absorption coefficient $\beta$, when the sample is optically opaque, i.e., $\beta \cdot d \gg 1$ and the optical penetration depth is smaller than the thermal diffusion length, i.e., $\beta^{-1}<\mu$, Eq. 2 can be presented as:

$$
T_{\mathrm{sat}}=\frac{\left(1-R_{\mathrm{opt}}\right)(1+R \cdot \exp (-2 \sigma d))}{\lambda \sigma(1-R \cdot \exp (-2 \sigma d))} .
$$

It is the case of the saturation of the temperature $T_{\text {sat }}$. The normalized amplitude of the PA signal is defined as:

$$
q=\left|\frac{P}{P_{\mathrm{sat}}}\right|=\left|\frac{T}{T_{\mathrm{sat}}}\right|
$$

where $P$ is the periodical component of the gas pressure in the PA chamber, $P_{\text {sat }}$ is the periodical component of the gas pressure in the PA chamber for the large value of the optical absorption coefficient.

The normalized amplitude of the PA signal can be expressed by Eq. 5 .

$$
q=\left|\frac{\beta}{1+R \cdot \exp (-2 \sigma d)}\left(\frac{1-\exp (-(\beta+\sigma) d)}{\beta+\sigma}\right)+\frac{R \cdot \exp (-2 \sigma d) \cdot(1-\exp (-(\beta-\sigma) d))}{\beta-\sigma}\right|
$$

The problem is that, in a general case, it is not possible to extract the value of the optical absorption coefficient $\beta$ from the value of $q$ in the analytical form from Eq. 5 . It can be computed only with the numerical methods.

For thin layers however, Eq. 5 can be transformed to a much simple form if the following conditions are fulfilled for the sample: $1 / \beta<\mu$ and $d / \mu \ll 1$ and the optically transparent substrate is thermally thick. The first condition means that the optical penetration depth $1 / \beta$ of the sample is smaller than its thermal diffusion length $\mu$. The second condition means that the sample is thermally thin.

Then, Eqs. (2), (3) and (5) can be presented as (6-8), respectively:

$$
\begin{gathered}
T=\frac{1-\exp (-\beta d)}{\lambda \sigma(1-R)}(1+R) \\
T_{\text {sat }}=\frac{\left(1-R_{\mathrm{opt}}\right) \cdot(1+R)}{\lambda \sigma(1-R)} \\
q=1-\exp (-\beta \cdot d) .
\end{gathered}
$$

In this simple model, the value of the optical absorption coefficient $\beta$ can be determined from the value of the normalized PA amplitude $q$ by Eq. 9 .

$$
\beta=-\frac{\ln (1-q)}{d} .
$$


Fig. 1 a Theoretical normalized PA amplitude spectrum $q$ (thick solid line) computed with Eq. 5. The parameters taken for computation are: $d=0.5 \mu \mathrm{m}$, $\alpha=0.01 \mathrm{~cm}^{2} \cdot \mathrm{s}^{-1}, f=36 \mathrm{~Hz}$, and the optical absorption coefficient spectrum was given by Eq. 10. b Optical absorption coefficient spectra: thick solid line-given by Eq. 10, thin solid lines-computed with formula (1) for thermal diffusivities $\alpha=0.001,0.01,0.1 \mathrm{~cm}^{2} \cdot \mathrm{s}^{-1}$, respectively. Circles, in Fig. 1a, are normalized amplitude data taken for computation, and in Fig. 1b, are optical absorption coefficient values computed with Eq. 9 in a model of thin solid sample
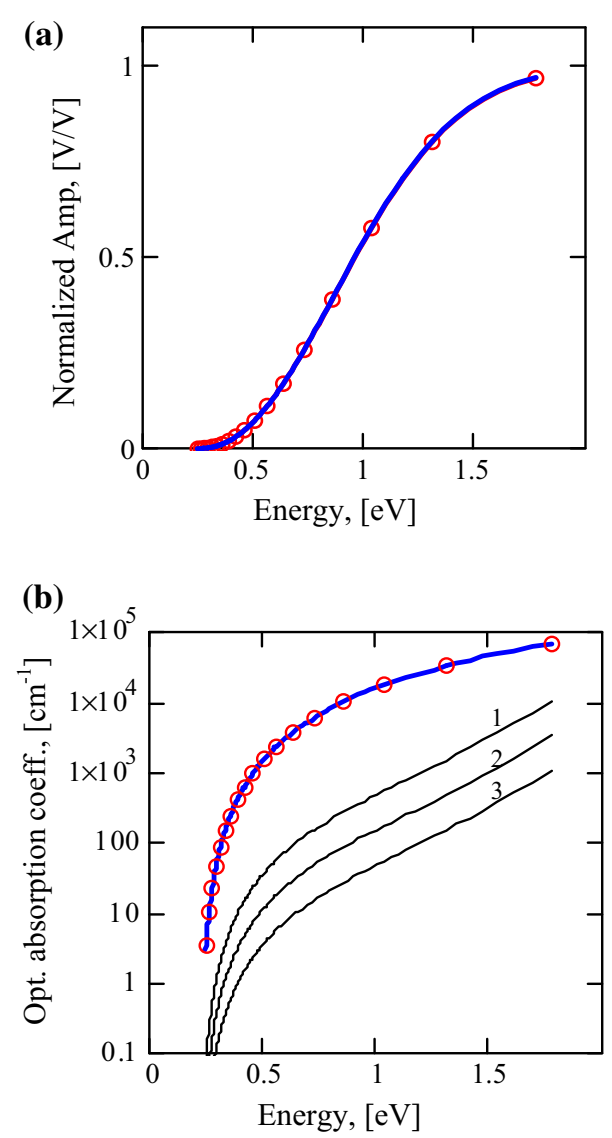

Let us consider theoretically an example thin semiconductor layer of the thickness $d=0.5 \mu \mathrm{m}$, deposited on a thermally thick transparent substrate. The thermal diffusivity of this layer is $\alpha=0.01 \mathrm{~cm}^{2} \cdot \mathrm{s}^{-1}$, and its optical absorption coefficient spectrum is given by Eq. 10. Frequency of the modulation of the intensity of the illuminating light in the PA simulation, taken for computations, $f=36 \mathrm{~Hz}$. Thermal diffusion length equals to $\mu=90 \mu \mathrm{m}$.

$$
\beta(h v)=3.33 \cdot 10^{4} \cdot \frac{1}{h v} \cdot(h v-0.22)^{3} .
$$

This one Eq. 10 describes well the optical absorption coefficient spectrum of a semiconductor sample with indirect electron type transitions for photon energies $h v$ larger than the energy band gap, and the Urbach absorption tail for $h v$ below the energy band gap.

The results of computations of the normalized PA amplitude spectrum and the optical absorption coefficient spectra are presented in Fig. 1a, b, respectively.

When we take the values of the q parameter, marked as circles in Fig. 1a, from the spectrum of the normalized PA amplitude computed in a full model (Eq. 5) (solid line 
Fig. 2 Spectrum of the normalized PA amplitude $q$ computed by Eq. 5 for different values of the thermal reflection coefficient $R$ between the thin sample and the glass substrate

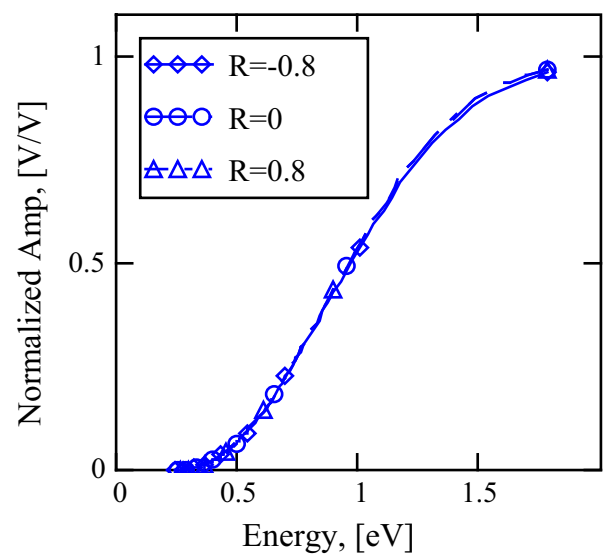

in Fig. 1a) and compute the corresponding values of the optical absorption coefficient $\beta$ in a simplified model (Eq. 9), we get values of $\beta$ presented as circles in Fig. 1b. One can see a perfect fit of the values of $\beta$ computed with a simplified model (Eq. 9), and assumed, theoretical absorption coefficient spectrum computed with Eq. 10 (thick solid line in Fig. 1b).

Optical absorption coefficient spectra computed by Eq. 1, in a model of a thermally and optically thick sample for different values of thermal diffusivities, are presented as thin lines in Fig. 1b. They show that such computed optical absorption coefficient spectra are incorrect.

The conditions of the correctness of this approach: $d / \mu \ll 1$ and $1 / \beta<\mu$ are fulfilled in the whole optical range presented in Fig. 1.

The dependence of the normalized photoacoustic amplitude $q$ on the thermal reflection coefficient $R$ between the thin layer and a transparent substrate is presented in Fig. 2. As one can see from this figure, the normalized PA amplitude $q$ does not depend on the value of the parameter $R$. It means that the normalized PA amplitude spectrum is independent of the thermal parameters of the transparent backing. What is more, the normalized PA amplitude spectrum is independent of the thermal parameters of the thin layer.

\subsection{Multiple Reflections of Light in the Sample can not be Neglected}

\subsubsection{Different Values of the Optical Reflections Coefficients}

The temperature of the front (illuminated) surface of the sample is given by equation:

$$
T=\frac{\left(1-R_{1}\right)\left(T_{F}+R_{2} \exp (-\beta d) T_{R}\right)}{\left(1-R_{1} R_{2} \cdot \exp (-2 \beta d)\right)}
$$

where $R_{1}$ is the optical reflection coefficient of the interface air-layer, $R_{2}$ is the optical reflection coefficient of the interface layer-glass backing, $T_{F}, T_{R}$ are the temperatures 
of the illuminated side of the layer (Front) and the opposite side of the layer (Rear) when optical reflections coefficients $R_{1}=R_{2}=0$.

Equation (11) is extended for two different values of the optical reflection coefficients, of the model presented in [17].

$T_{F}$ and $T_{R}$ are given by equations:

$$
\begin{gathered}
T_{F}=\frac{\beta}{\lambda \sigma(1-R \cdot \exp (-2 \sigma d))}\left(\frac{1-\exp (-(\beta+\sigma) d)}{\beta+\sigma}+\frac{R \cdot \exp (-2 \sigma d) \cdot(1-\exp (-(\beta-\sigma) d))}{\beta-\sigma}\right) \\
T_{\mathrm{R}}=\frac{\beta \exp (-\sigma d)}{\lambda \sigma(1-R \cdot \exp (-2 \sigma d))}\left(\frac{R \cdot(1-\exp (-(\beta+\sigma) d))}{\beta+\sigma}+\frac{1-\exp (-(\beta-\sigma) d)}{\beta-\sigma}\right)
\end{gathered}
$$

when the conditions $\beta \gg 1 / \mu$ and $d / \mu \approx 0$ are fulfilled, one gets:

$$
\begin{gathered}
T_{F}=\frac{(1-\exp (-\beta d))(1+R \cdot \exp (-2 \sigma d))}{\lambda \sigma(1-R \cdot \exp (-2 \sigma d))}=\frac{(1-\exp (-\beta d))(1+R)}{\lambda \sigma(1-R)} \\
T_{R}=\frac{(1-\exp (-\beta d))(1+R) \exp (-\sigma d)}{\lambda \sigma(1-R \cdot \exp (-2 \sigma d))}=\frac{(1-\exp (-\beta d))(1+R)}{\lambda \sigma(1-R)} .
\end{gathered}
$$

Then, for this case $T_{F}=T_{R}$ and the temperature $T$ is given by Eq. 16 .

$$
T=\frac{\left(1-R_{1}\right)\left(1+R_{2} \exp (-\beta d)\right)}{\left(1-R_{1} R_{2} \cdot \exp (-2 \beta d)\right)} T_{F}=\frac{\left(1-R_{1}\right)\left(1+R_{2} \exp (-\beta d)\right)}{\left(1-R_{1} R_{2} \cdot \exp (-2 \beta d)\right)} \frac{(1-\exp (-\beta d))(1+R)}{\lambda \sigma(1-R)}
$$

$T$ for the large optical absorption coefficient $\beta$, when $\beta d \gg 1$ is denoted as $T_{\text {sat }}$ and is given by Eq. 17 .

$$
T_{\mathrm{sat}}=\frac{\left(1-R_{1}\right)(1+R)}{\lambda \sigma(1-R)} .
$$

Then, the normalized PA amplitude $q$ is given by Eq. 18 .

$$
q=\frac{\left(1+R_{2} \exp (-\beta d)\right)(1-\exp (-\beta d))}{\left(1-R_{1} R_{2} \exp (-2 \beta d)\right)} .
$$

\subsubsection{Equal Values of the Optical Reflections Coefficients $R_{1}$ and $R_{2}$}

In this case, the temperature $T, T_{\text {sat }}$ and the normalized PA amplitude $q$ are given by Eqs. (19-21), respectively. Equal values of the optical reflection coefficients $R_{1}$ and $R_{2}$ are denoted as $R_{\mathrm{O}}$.

$$
\begin{gathered}
T=\frac{\left(1-R_{o}\right)\left(1+R_{0} \exp (-\beta d)\right) T_{F}}{\left(1-R_{o}^{2} \exp (-2 \beta d)\right)}=\frac{\left(1-R_{o}\right)\left(1+R_{o} \exp (-\beta d)\right)}{\left(1-R_{o}^{2} \cdot \exp (-2 \beta d)\right)} \frac{(1-\exp (-\beta d))(1+R)}{\lambda \sigma(1-R)} \\
T_{\mathrm{sat}}=\frac{\left(1-R_{o}\right)(1+R)}{\lambda \sigma(1-R)} \\
q=\frac{\left(1+R_{o} \exp (-\beta d)\right)(1-\exp (-\beta d))}{\left(1-R_{o}^{2} \exp (-2 \beta d)\right)}=\frac{1-\exp (-\beta d)}{1-R_{o} \exp (-\beta d)}
\end{gathered}
$$


Fig. 3 a Normalized PA

amplitude q for sample with $R_{\mathrm{O}}$ $=0$ (dashed line) and $R_{\mathrm{O}}=0.5$

(solid line) computed by Eqs. 11 and 10. b The optical absorption coefficient spectra computed by Eq. 22 for different values of $R_{\mathrm{O}}$ $=0,0.4$ and 0.9 (diamonds, circles, squares, respectively). A thick solid line is the optical absorption coefficient characteristics used for calculation of $q$ given by Eq. 10

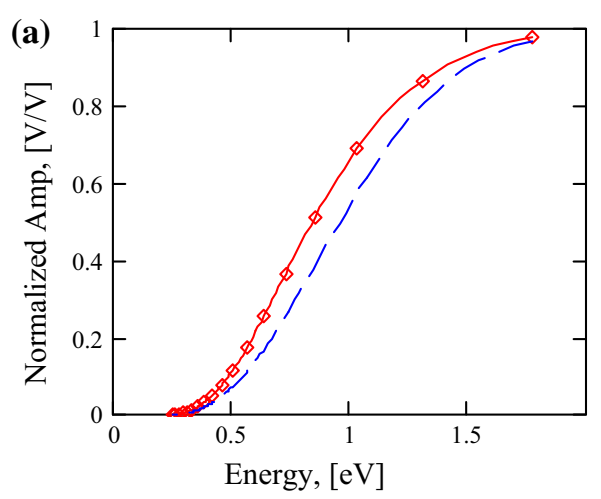

(b)

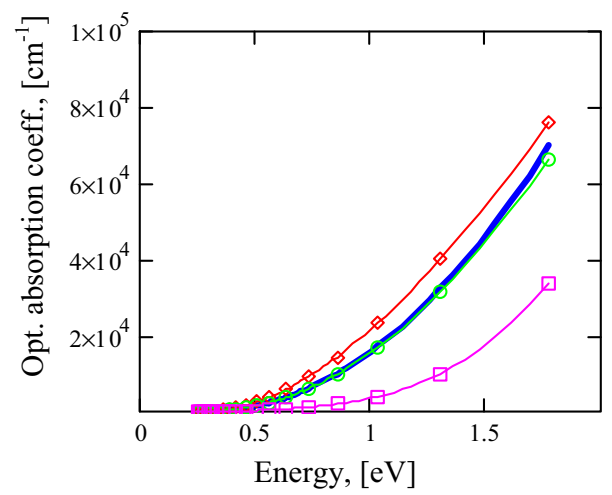

In this case, the optical absorption coefficient can be computed from the normalized PA amplitude by equation:

$$
\beta=-\frac{1}{d} \ln \left(\frac{1-q}{1-R_{o} q}\right)
$$

The influence of the value of the optical reflection coefficient on the normalized PA amplitude spectrum and on the computed optical absorption coefficient spectrum is presented in Fig. 3.

As one can see, when the optical reflection coefficient $R_{o}$ of the sample is known, the optical absorption coefficient spectrum computed by Eq. 22, from the normalized PA amplitude spectrum, fits very well to the assumed optical absorption coefficient spectrum. Comparison of the results of computations of the optical absorption spectra by Eqs. 9 and 22 shows that for thin semiconductor samples when $R_{o}$ is in the range of $0.2-0.36$ the error of computations of the optical absorption coefficient spectra is smaller than $15 \%$. 
Fig. 4 Experimental spectra of the normalized photoacoustic amplitude $q$ obtained for two thin Ge samples, i.e., S1 (diamonds) and S2 (circles). Frequency of modulation $f=$ $36 \mathrm{~Hz}$

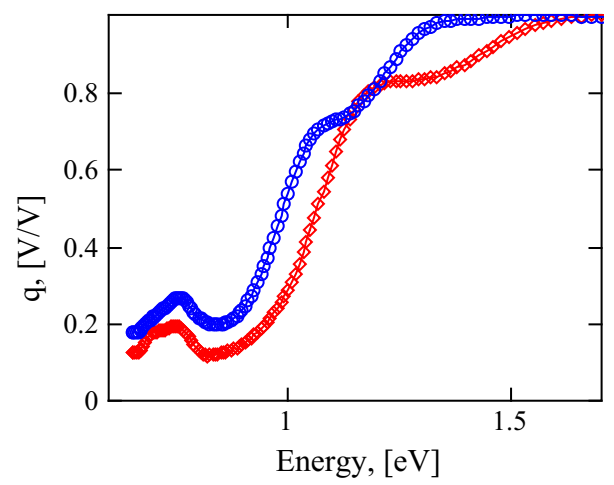

\section{Experimental Results}

The method described above was next applied for investigations of the optical absorption coefficient spectra of thin germanium layers evaporated on a glass substrate. The experimental normalized amplitude photoacoustic spectra of germanium samples are presented in Fig. 4.

Thicknesses of germanium layers were determined from the positions of the interference maxima on the normalized photoacoustic amplitude spectra, using Eq. 23. This equation can be found elsewhere, e.g., [24, 25].

$$
d=\frac{1}{2 n}\left(\frac{p \lambda_{m} \lambda_{m+p}}{\lambda_{m}-\lambda_{m+p}}\right)
$$

where $\lambda_{m}, \lambda_{m+p}$ are the wavelengths of the $m$ th and $(m+p)$ th minima/maxima, $p$ is the number of oscillations between the two extrema ( $p=1$ between two consecutive minima or maxima); $n$ is the refractive index, assumed as a constant between the extrema used for calculations.

For sample $\mathrm{S} 1 \lambda_{m}=1050 \mathrm{~nm}, \lambda_{m+p}=1680 \mathrm{~nm}, p=1$,

For sample S2 $\lambda_{m}=1180 \mathrm{~nm}, \lambda_{m+p}=1640 \mathrm{~nm}, p=1$,

The thickness $d_{1}=320 \mathrm{~nm}$ of S1 sample and thickness $d_{2}=492 \mathrm{~nm}$ of S2 sample were determined by Eq. 23.

The optical absorption coefficient spectrum of germanium layers is presented in Fig. 5 as a thick solid line.

The optical absorption coefficient spectra of germanium layers were computed by Eq. 9.

The Tauc plot characteristics, $(\beta \cdot h v)^{0.5}$ versus the energy of photons $h v$, for the optical absorption coefficient spectrum of a thin germanium layer are presented in Fig. 6.

The solid line represents the characteristics for the indirect electron type transitions of type $\beta \cdot h v=A \cdot\left(h v-E_{g}\right)^{2}$. From the fitting of theoretical curve to the experimental data, the parameters $A=3 \cdot 10^{5} \mathrm{eV}^{-1} \cdot \mathrm{cm}^{-1}$ and $E_{\mathrm{g}}=0.79 \mathrm{eV}$ were determined for the energy of photons $h v>0.8 \mathrm{eV}$. 
Fig. 5 Optical absorption coefficient spectra of thin germanium layers, and the average optical absorption coefficient spectrum. Diamonds and circles are computed data for samples $\mathrm{S} 1$ and $\mathrm{S} 2$, respectively. The thick solid line is the average optical absorption coefficient spectrum of the thin germanium layers

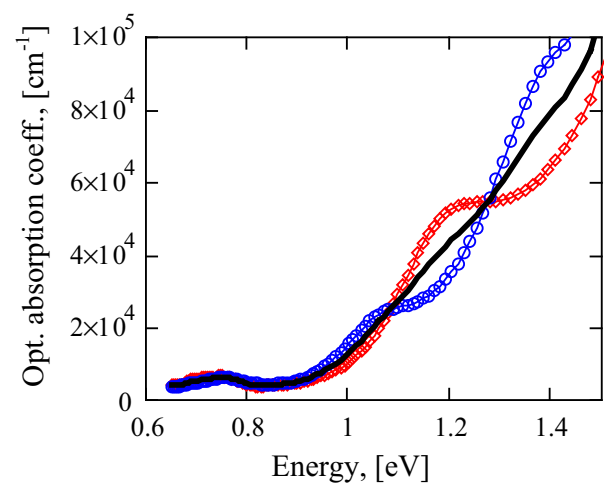

Fig. 6 The Tauc plot

characteristics of the average optical absorption coefficient spectrum of a thin germanium layer for energies of photons above $0.8 \mathrm{eV}$

Fig. 7 Comparison of the optical absorption coefficient spectra of thin germanium layers, obtained with the photoacoustic method (solid line), and the literature optical absorption spectra, shown in references [26] (circles) and Ref. [27] (diamonds), for monocrystal and amorphous germanium, respectively
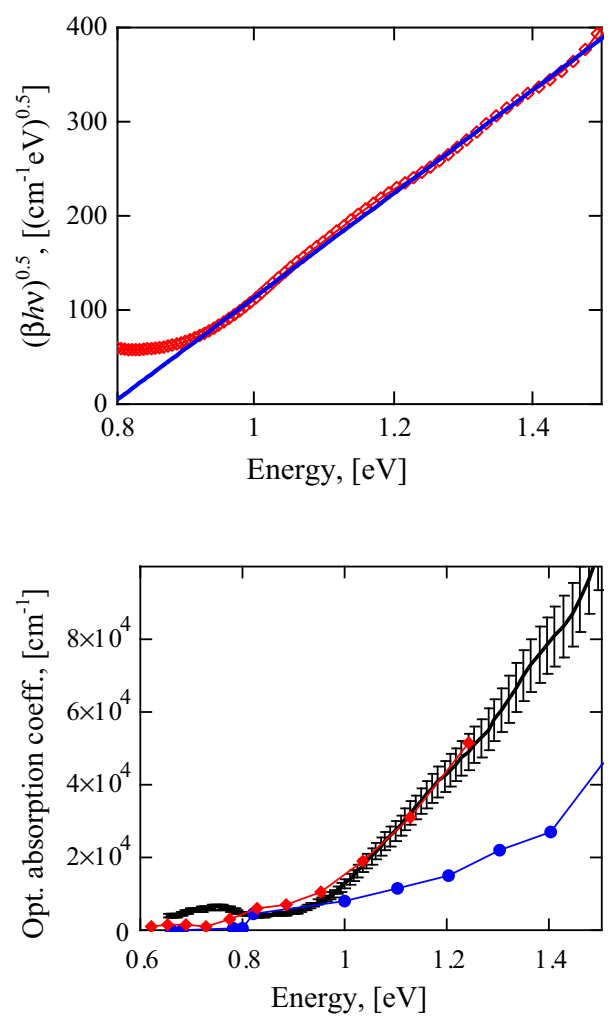

The comparison of the experimental optical absorption coefficient spectrum, obtained for germanium samples with the presented photoacoustic method, and the literature data is presented in Fig. 7.

The results showed in references [26] and [27] were obtained with optical reflectance and transmittance methods for monocrystal and amorphous germanium samples, respectively. 


\section{Conclusions}

This paper presents the new practical equations for computations of the optical absorption coefficient absorption spectra from the experimental normalized PA amplitude spectra. The equations were derived under the several conditions. The thin semiconductor film is deposited on the optically transparent and thermally thick substrate. It is thermally thin, and its optical penetration depth in the thin layer is smaller than its thermal diffusion length. The literature analysis shows that these conditions are fulfilled for majority of investigated thin films deposited on the glass substrates. This model applied for investigations of thin germanium layers deposited on the transparent glass substrate proved its correctness and usefulness. The obtained experimental optical absorption coefficient spectrum of a thin germanium layer is in a good agreement with literature [27] spectrum of thin amorphous germanium layers obtained with the purely optical methods.

Open Access This article is distributed under the terms of the Creative Commons Attribution 4.0 International License (http://creativecommons.org/licenses/by/4.0/), which permits unrestricted use, distribution, and reproduction in any medium, provided you give appropriate credit to the original author(s) and the source, provide a link to the Creative Commons license, and indicate if changes were made.

\section{References}

1. E. Ahmed, A. Zegadi, A.E. Hill, R.D. Pilkington, R.D. Tomlinson, Thin Solid Films 268, 144-151 (1995)

2. K.T. Ramakrishna Reddy, R.B.V. Chalapathy, M.A. Slifkin, A.W. Weiss, R.W. Miles, Thin Solid Films 387, 205-207 (2001)

3. H.W. Schock, Sol. Energy Mater. Sol. Cells 34, 19-26 (1994)

4. V.F. Gremenok, I.A. Victorov, L.G. Beresutski, E.P. Zaretskaya, M.V. Yakushev, in Ternary and Multinary Compounds, ed. by R.D. Tomlinson, A.E. Hill, R.D. Pilkington (CRC Press, London, 1998), p. 217

5. O. Vigil-Galan, E. Marin, J. Sastre Hernandez, E. Saucedo, C.M. Ruiz, G. Contreras-Puente, A. Calderon, J. Mater. Sci. 42, 7176-7179 (2007)

6. M.L. Albor-Aguilera, M.A. Gonzalez-Trujillo, A. Cruz-Orea, M. Tufino-Velzaguez, Thin Solid Films 517, 2335-2339 (2009)

7. A. Fernandez-Perez, M.G. Sandoval-Paz, R. Saavedra, Chalcogenide Lett. 13, 507-514 (2016)

8. P. Raji, K. Ramachandran, C. Sanjeeviraja, J. Mater. Sci. 41, 5907-5914 (2006)

9. S. Rasool, K. Saritha, K.T. Ramakrishna Reddy, K. Raveendranath Reddy, L. Bychto, A. Patryn, M. Maliński, M.S. Tivanov, V.F. Gremenok, Mat. Sci. Semicon. Proc. 72, 4-8 (2017)

10. N.G.C. Astrath, A.C. Bent, M.L. Baesso, A. Ferreira da Silva, C. Persson, Thin Solid Films 515, 2821-2823 (2006)

11. K. Jeyadheepan, P. Palanichamy, V. Swaminathan, M. Jayachandran, C. Sanjeeviraja, Appl. Phys. A 98, 919-925 (2010)

12. M. Malinski, Ł. Chrobak, L. Bychto, Solid State Commun. 150, 424-427 (2010)

13. M. Malinski, Ł. Chrobak, Opto Electron. Rev. 18, 190-196 (2010)

14. M. Maliński, L. Bychto, S. Łęgowski, J. Szatkowski, J. Zakrzewski, Microelectron. J. 32, 903-910 (2001)

15. M. Maliński, Ł. Chrobak, Opto Electron. Rev. 19, 46-50 (2011)

16. H. Neuman, Crys. Res. Technol. 28, 73-82 (1993)

17. M. Maliński, Opto-Electron. Rev. 18, 474-477 (2010)

18. M.A. Zambrano-Arjona, J. Ordonez-Miranda, R.A. Medina -Esquivel, F. Penunuri, P. Martinez, J.J. Alvarado-Gil, J. Appl. Phys. 111, 094915 (2012)

19. H. Benamrani, F.Z. Satour, A. Zagadi, A. Zouaoui, J. Lumin. 132, 305-312 (2012) 
20. P. Poulet, J. Chambron, R. Unterreiner, J. Appl. Phys. 51, 1738-1740 (1980)

21. J. Fresquet, B. Girault, D.M. Razafindrandriatsimaniry, Appl. Opt. 23, 2784-2787 (1984)

22. A.K. Gosh, K.K. Som, S. Chatterjee, B.K. Chandhuri, Phys. Rev. B 51, 4842-4848 (1995)

23. M. Maliński, Arch. Acoust. 27, 217-228 (2002)

24. J.C. Manifacier, J. Gasiot, J.P. Fillard, J. Phys. E: Sci. Instrum. 9, 1002-1004 (1976)

25. R. Swanepoel, J. Phys. E: Sci. Instrum. 16, 1214-1222 (1983)

26. R.F. Potter, in Handbook of Optical Constants of Solids, ed. by E.D. Palik (Academic Press, New York, 1985), p. 465

27. T.M. Donovan, W.E. Spicer, J.M. Benett, E.J. Ashley, Phys. Rev. B 2, 397-413 (1970) 